\title{
Subarachnoid hemorrhage and hydrocephalus causing neurogenic pulmonary edema
}

\author{
Eberval Gadelha Figueiredo', Arthur Maynart Pereira Oliveira², \\ Carlos Eduardo Romeu de Almeida ${ }^{3}$, Manoel Jacobsen Teixeira ${ }^{4}$
}

Neurogenic pulmonary edema (NPE) is described as the acute onset of dyspnea or a decrease in $\mathrm{PaO} 2 / \mathrm{FiO} 2$ ratio caused by a pulmonary interstitial and alveolar congestion after an acute central nervous system (CNS) damage, without obvious cause of lung injury ${ }^{1-3}$. However, NPE may be also caused by myocardial failure ${ }^{4}$ and has been reported in the chronic setting of terminal cerebral tumors ${ }^{5}$. In spite of its high frequency, NPE is an underdiagnosed, life-threatening and often overlooked condition ${ }^{6}$.

We describe a case of a patient sustaining subarachnoid hemorrhage (SAH) and hydrocephalus that developed NPE.

\section{CASE}

A 52 years-old female without pre-existing cardiopulmonary disease presented at the emergency room with a history of sudden headache followed by loss of conscience. At physical examination, she was alert, but confuse, with no abnormalities on the neurological exam. CT scan displayed a Fischer grade III SAH. Four hours after, she became lethargic, with tachycardia and dyspnea. Chest radiograph revealed bilateral alveolar infiltrates (Fig 1). The electrocardiogram (ECG) was unchanged. Mild hemoptysis was also observed and patient underwent orotraqueal intubation and diuretics were administered. Repeat CT scan revealed increase in the size of the ventricles (Fig 2) and a ventriculostomy was emergentially performed.

Soon after, patient recovered completely and presented significant improvement

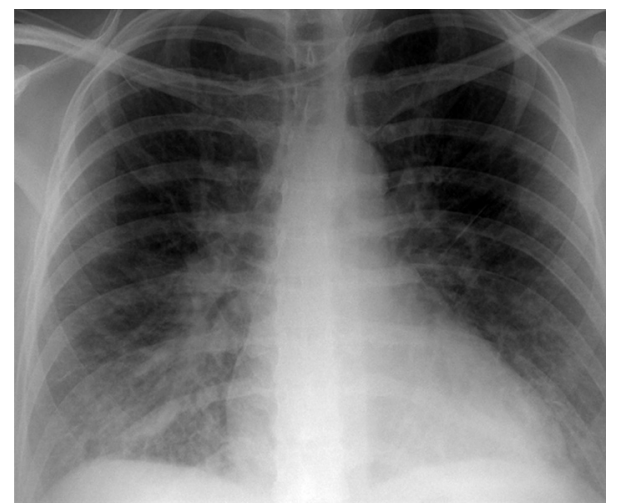

Fig 1. Chest radiograph revealed bilateral alveolar infiltrates.

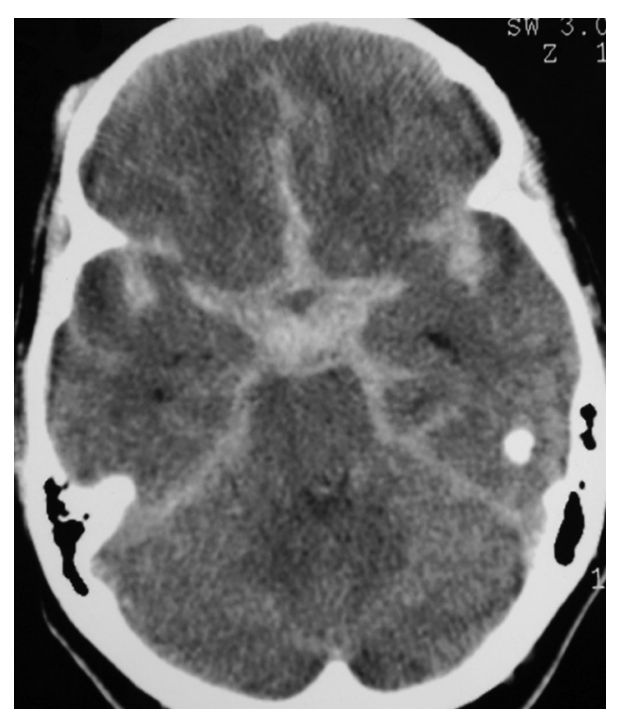

Fig 2. CT scan revealed a subrachnoid hemorrage.

in the radiological and functional parameters. On the following day, she underwent microsurgical clipping of her aneurysm without complications. Her relatives have given permission to publish this case. 


\section{DISCUSSION}

The most important cause of NPE is subarachnoid hemorrhage ${ }^{1,7,8}$ followed by cerebral trauma ${ }^{9,10}$ and epilep$\mathrm{sy}^{1}$. Other less common causes may include cervical spine trauma, meningitis, multiple sclerosis, cerebellar hemorrhage, cerebral gas embolism, intracranial tumors ${ }^{5}$ and ventricular shunt dysfunction.

The incidence of NPE after SAH in the literature may vary from 4 to $23 \%$ in greater studies ${ }^{6}$, with $\mathrm{SAH}$ accounting for $43-73 \%$ of all cases of NPE. The most important factors for NPE development after SAH are the clinical and radiological severity of bleeding, as well as posterior circulation bleedings ${ }^{1}$. NPE arises more often between the first and seventh day after SAH, associated with primary or secondary cerebral insults ${ }^{1}$. NPE has also been reported in up to $20 \%$ of cases of severe head injury (Glasgow Coma Scale <8).

It is postulated that NPE may be a consequence of two mechanisms: an excessive adrenergic discharge which leads to pulmonary vasoconstriction and a rapid increase in pulmonary capillary hydrostatic pressure, thus causing fluid leakage to the alveolar space. A hydrostatic edema appears when transmural pressure exceeds $40 \mathrm{mmHg}$. Such raise in hydrostatic pressure may damage or induce an inflammatory response to the endothelium and basement membrane, leading to protein leakage and promoting the alveolar exudate typically seen in $\mathrm{NPE}^{1-3}$.

The anatomic sites implicated in the autonomic regulation of pulmonary capillary tonus beingresponsible for NPE are the posterior hypothalamus, ventral medulla, including the A1 catecholaminergic neurons, posterior medulla and cervical spinal chord ${ }^{1-3}$. It is most likely that any condition damaging the anatomical areas responsible for pulmonary capillary tonus control may lead to the development of various degrees of NPE. The acute onset of cerebral hypertension and chemical irritation may be important underlying factors, both present in SAH. Also, the rapidity of increase in intracranial pressure, and not intracranial hypertension alone seems to be responsible for NPE. This fact may explain the appearance of NPE after hydrocephalus that was observed in this case.

NPE shows a broad clinical spectrum, ranging from the asymptomatic patient to the rapid development of respiratory failure. It typically presents within minutes to hours from a severe CNS insult, with the acute onset of dyspnea or a decrease in the $\mathrm{PaO} 2 / \mathrm{FiO} 2 \mathrm{ratio}^{3}$. Chest radiograph classically shows bilateral alveolar infiltrates, as seen in this case, but unilateral NPE is also possible. Echocardiography, transesophageal Doppler, electrocardiogram and central venous pressure values are normal. Nonetheless, NPE may be provoked by neurogenic myocardial failure or a pre-existing cardiopulmonary disease. Pathologically high cardiac biomarkers without cardiac dysfunction may be present in up to $83 \%$ of patients with $\mathrm{NPE}^{6}$. Although rarely assessed, estimation of pulmonary capillary pressure may be a better method to assess induced capillary changes in NPE.

Differential diagnosis with NPE should include aspiration pneumonitis, a frequent condition in neurological patients, ventilator-associated pneumonia and ventilation induced lung injury ${ }^{1}$. The blood level of pro-calcitonin may provide evidence of invasive bacterial infection. The absence of suspicion of aspiration during tracheal intubation and the appearance of tracheal secretions, the rapid development of pulmonary edema, absence of fever, and rapid resolution of symptoms are suggestive of NPE. Thus, NPE is a retrospective diagnosis in many cases. Definitive diagnosis of NPE is difficult because of the nonspecific nature of clinical signs and routine diagnostic tests. A clinical diagnosis of NPE is based largely upon the occurrence of pulmonary edema in the appropriate setting and in the absence of another obvious cause as occurred in the present case ${ }^{3}$.

NPE is generally treated in a supportive and conservative fashion ${ }^{3}$, and patient management should be focused in the primary disease. Non-invasive ventilation should be preferred whenever possible. Invasive ventilation should be used cautiously because of the risk of diminished venous return and increase in $\mathrm{ICP}^{1,3}$. Permissive hypercapnia and prone positioning should be allowed only if ICP monitoring is available ${ }^{1 .}$ Extracorporeal life support and hypothermia are alternative treatments .Cardiac dysfunction may be present in several cases and care must be taken to reduce pre and after-load ${ }^{1,3}$ and to increase cardiac contractility. Experimental data suggest that the use of alphaadrenergic blockers can hasten the resolution of $\mathrm{NPE}^{3}$.

The pure form of NPE may resolve within 48-72h with adequate treatment ${ }^{1,3}$. The patient's prognosis generally depends on the neurological condition. Overall mortality in NPE is estimated in $7-10 \%{ }^{2,8}$.

\section{REFERENCES}

1. Baumann A, Audibert G, McDonnel J, Mertes P. Neurogenic pulmonary edema. Acta Anaesthesiol Scand 2007:51:447-455.

2. Sedý J, Zicha J, Kunes J, Jendelová P, Syková E. Mechanisms of neurogenic pulmonary edema development. Physiol Res 2008:57:499-506.

3. UpToDate. Available at: http://www.uptodateonline.com/online/index.do

4. Simon RP. Neurogenic pulmonary edema. Neurol Clin 1993;11:309-323.

5. Macleod AD. Neurogenic pulmonary edema in palliative care. J Pain Symptom Manage 2002;23:154-156.

6. Muroi C, Keller M, Pangalu A, Fortunati M, Yonekawa Y, Keller E. Neurogenic pulmonary edema in patients with subarachnoid hemorrhage. J Neurosurg Anesthesiol 2008;20:188-192.

7. Shanahan WT. Acute pulmonary edema as a complication of epileptic seizures. NY Med J 1908;37: 54-56.

8. Fontes RB, Aguiar PH, Zanetti MV, Andrade F, Mandel M, Teixeira MJ. Acute neurogenic pulmonary edema: case reports and literature review. J Neurosurg Anesthesiol 2003;15:144-150.

9. Dettbarn CL, Davidson LJ. Pulmonary complications in the patient with acute head injury: neurogenic pulmonary edema. Heart Lung 1989;18:583-589.

10. Rogers FB, Shackford SR, Trevisani GT, Davis JW, Mackersie RC, Hoyt DB. Neu rogenic pulmonary edema in fatal and nonfatal head injuries. J Trauma 1995; 39:860-866 